\title{
Solitary fibrous tumor of the pleura mimicking neurogenic tumor
}

\author{
Giuseppe De Luca, MD, ${ }^{a}$ Salvatore Griffo, MD, ${ }^{a}$ Ciriaco Cecere, MD, ${ }^{a}$ Marcellino Cicalese, MD, ${ }^{a}$ Luigi Di Tommaso, MD, \\ Mario Monaco, MD, ${ }^{\mathrm{b}}$ and Paolo Stassano, MD, ${ }^{\mathrm{b}}$ Naples, Italy
}

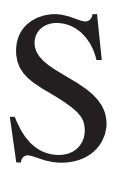

olitary fibrous tumor (SFT) is a rare tumor affecting adults. The incidence of SFT is 2.8 cases per 100,000, and it is commonly located in the visceral and parietal pleura. ${ }^{1}$ Extrapleural sites also have been described. ${ }^{2}$ In contrast to malignant mesothelioma, it occurs independently of asbestos exposure. The diagnosis of SFT requires immunohistologic analysis because of its lack of distinctive histologic characteristics: the "patternless pattern."3

We report an SFT located in the mediastinum that mimicked a neurogenic tumor.

\section{Clinical Summary}

A 55-year-old woman was admitted to our hospital with increasing symptoms of dyspnea and cough without sputum production. She denied any history of cigarette smoking and any asbestos exposure. The results of routine blood testing, electrocardiography, spirometry, and arterial blood gas analysis were normal. Chest radiography revealed a well-circumscribed round mass in the left superior mediastinum. A chest computed tomographic (CT) scan confirmed a well-delineated mass of solid appearance in the superior left mediastinum, but the pleural relationship was unclear. This mass measured $6 \times 6.5 \mathrm{~cm}$,

From the Thoracic ${ }^{\mathrm{a}}$ and Cardiac $^{\mathrm{b}}$ Surgery Units, University Federico II, Naples, Italy.

Received for publication June 7, 2006; accepted for publication June 15, 2006.

Address for reprints: Giuseppe De Luca, MD, C.so Secondigliano, 519, 80144 Naples, Italy (E-mail: giudeluc@unina.it).

J Thorac Cardiovasc Surg 2006;132:1241-2

$0022-5223 / \$ 32.00$

Copyright (๑) 2006 by The American Association for Thoracic Surgery doi:10.1016/j.jtcvs.2006.06.035 involved the costovertebral plane and the aortic arch, and displaced the adjacent lung parenchyma, thus simulating a peripheral neurogenic tumor (Figure 1, A). Subsequently, magnetic resonance imaging was carried out: there was no aortic arch involvement, but the mass was indissociable from the rachis, and the suspicion of a neurogenic tumor could not be ruled out (Figure 1,B). A CT-guided needle aspiration of the mass yielded inconclusive results, and therefore surgical resection was planned. General anesthesia was induced, and the patient was intubated with an endotracheal double-lumen tube. With the patient in the right lateral decubitus position, a muscle-sparing lateral thoracotomy in the left fourth intercostal space was performed. The tumor was easily seen, originating from the parietal pleura with a vascularized pedicle. There was no invasion into the mediastinal structures. The mass was carefully isolated, and en bloc resection, with partial pleurectomy to minimize the chances of recurrence, was carried out. At gross examination, the mass measured $6 \times 6.5 \mathrm{~cm}$ and appeared firm, encapsulated, smoothly lobulated, and well circumscribed. The postoperative course was uneventful, and the patient was discharged home on postoperative day 6 .

Histologically, the lesion showed a tangled network of fibroblast-like cells squeezed in abundant collagen fibers, many of which were keloid-like and arranged in a patternless pattern. There was a lack of nuclear aberration and rare mitoses. There were no necrotic or hemorrhagic zones (Figure 2, A). Immunohistochemical staining showed strong positivity for vimentin, CD34, CD99, and blc-2 in fibroblast-like cells (Figure 2, B). A final diagnosis of benign fibrous tumor of the pleura was made. The specimen was sent to an outside reviewer at another institution, who confirmed the diagnosis.

One year later, the patient is in good health, and chest radiography and $\mathrm{CT}$ scanning have revealed no sign of local or distant tumor recurrence.

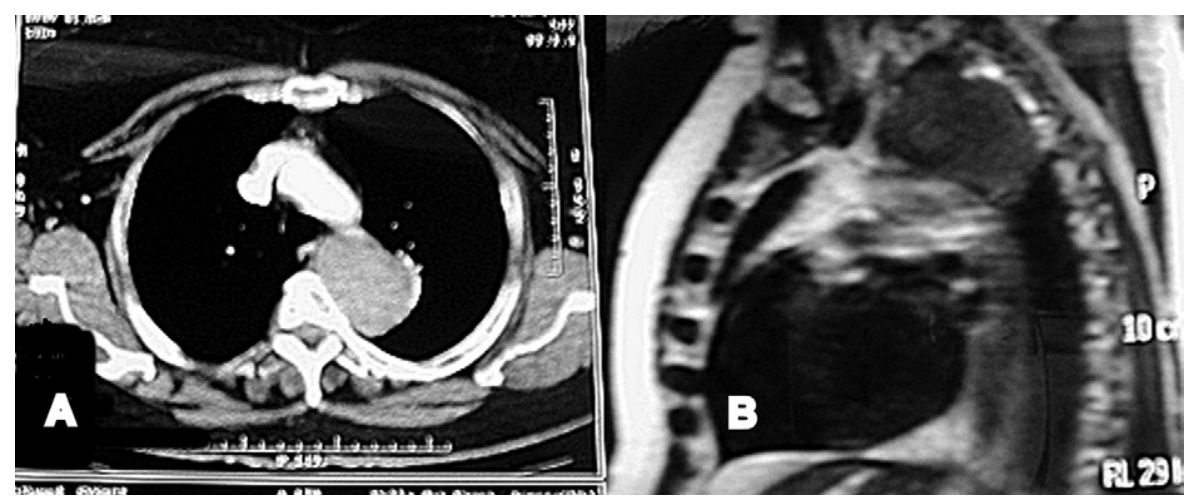

Figure 1. Chest computed tomographic scan $(A)$ and chest magnetic resonance image (B) showing a well-delineated mass mimicking a peripheral neurogenic tumor. 


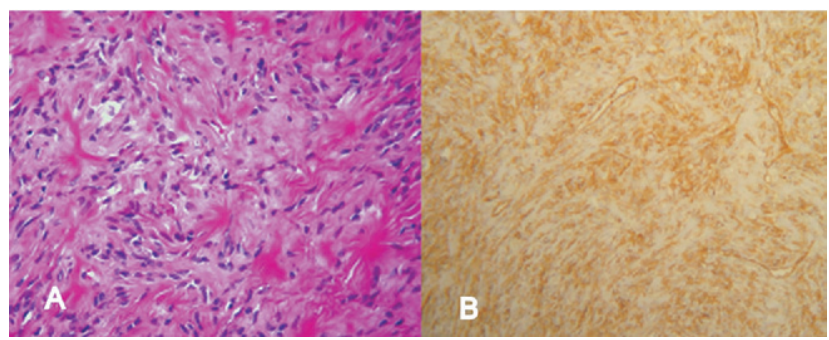

Figure 2. A, Tangled network of fibroblast-like cells squeezed in abundant collagen fibers and arranged in a patternless pattern. (Hematoxylin and eosin stain, original magnification $40 \times$.) B, Immunohistochemical stain shows strong positivity for CD34. (Original magnification $10 \times$.)

\section{Discussion}

The SFT is an uncommon spindle cell neoplasm of the pleura and was first described by Wagner in $1870 .{ }^{4}$ Klemper and Rabin ${ }^{5}$ interpreted the SFT as a form of mesothelioma and classified it in localized and diffuse types. SFT is a rare tumor, now proved to be of nonmesothelial origin, representing a small percentage of thoracic surgical activity $(<1 \%) .{ }^{1}$ It develops between the fifth and seventh decades of life, regardless of a previous history of smoking or asbestos exposure, and usually it is of a benign nature, with the malignant form occurring in $12 \%$ of cases. ${ }^{1,2}$ SFTs are usually of modest size, asymptomatic or poorly symptomatic, and occasionally discovered at routine chest radiography. Extrathoracic symptoms, seen in large tumors, include osteoarthropathy, digital clubbing, and hypoglycemia. ${ }^{2}$ The malignant form of SFT is bigger and symptomatic. ${ }^{2}$
Diagnostic tools, such as chest radiography, CT scanning, and magnetic resonance imaging, are helpful but not decisive in establishing the diagnosis. Even fine-needle aspiration is often inconclusive. Only surgical excision, with subsequent immunohistologic examination, can be diagnostic. Immunohistochemically positive CD34 staining is decisive in establishing the diagnosis differentiating SFT from mesotheliomas, synovial sarcomas, fibrosarcomas, and neurogenic tumors. In addition, the bcl-2 essay can confirm the diagnosis of SFT in case of CD34 negativity.

Complete surgical excision with underlying tissue removal lends itself to complete cure and minimizes tumor recurrence. Adjuvant postoperative chemotherapy, radiotherapy, or both have been used, but their efficacy remains unproved. Thoracoscopic surgery represents a valid and more modern approach, even if contact metastasis and local recurrence can occur with this technique. ${ }^{2}$

We believe that because of SFT's malignant potential and the uncertain diagnosis by means of needle or transbronchial biopsy, surgical exploration is imperative, even in asymptomatic patients.

\section{References}

1. Luckraz H, Ehsan S, Gibbs AR, Butchart EG. A giant pleural fibrous tumor. J Thorac Cardiovasc Surg. 2005;130:1216-7.

2. de Perrot M, Fischer S, Bründler M, Sekine Y, Keshavjee S. Solitary fibrous tumors of the pleura. Ann Thorac Surg. 2002;74:285-93.

3. Stout AP. Tumors of the pleura. Harlem Hosp Bull. 1971;5:54-7.

4. Wagner E. Das teberkelähnliche lymphadenom (Der cytogene oder reticulirte tuberkel). Arch Heilk (Leipzig). 1870;11:497.

5. Klemper P, Rabin CB. Primary neoplasm of the pleura: a report of five cases. Arch Pathol. 1931;11:385-412. 